\title{
Ischaemic heart disease and the proportions of hydrogenated fat and ruminant-animal fat in adipose tissue at post-mortem examination: a case-control study
}

\author{
LEO H. THOMAS AND R. G. SCOTT \\ From the Departments of Science and Mathematics/Computer Science, the Polytechnic of Wales, Pontypridd
}

SUMMARY Fatty acids characteristic of ruminant-animal fat have been found to be present in significantly lower proportions in samples from the depot fat of persons dying of ischaemic heart disease (cases) than in specimens from persons dying of unrelated causes (controls). Although such acids are also present, in lesser amounts, in hydrogenated marine oils, this case-versus-control difference is difficult to explain other than on the basis that controls consumed a higher proportion of ruminant-animal fat in their total dietary fat than did the cases.

The proportions of polyunsaturated acids and of certain higher $\left(\mathrm{C}_{20}\right.$ and $\mathrm{C}_{22}$ mostly mono-enoic) acids in the depot fat of cases and controls are virtually identical. There is also no indication of any difference in degrees of saturation of the fats between the case and control specimens.

Thomas ${ }^{1}$ showed that in Great Britain mortality from arteriosclerotic disease is highest in those areas and those social classes which consume the highest amounts of industrially hydrogenated fats (HF) and the lowest amounts of total butter fat.

We have demonstrated ${ }^{2}$ that subject to certain conditions the mean composition of depot fat in a sample drawn from a known population reflects in detail the composition of the fatty diet of that population except that dietary 16:0 and 18:0 acids are to some extent desaturated to $16: 1$ and 18:1 respectively.

We decided, therefore, to continue these studies and to examine whether the body fat of persons dying of ischaemic heart disease (IHD) contains a higher proportion of HF and a lower proportion of ruminant-animal fat (RAF) than the body fat of those dying of unrelated causes.

The analytical problem was to identify those particular fatty acids characteristic of HF and RAF, and whereas difficulties remain, transunsaturated acids and certain odd-numbered and branched-chain acids would appear to serve the purpose.

We have shown ${ }^{2}$ from analysis of British margarines purchased in 1976 that the hard materials contained on average about $50 \%$ hydrogenated marine animal or fish oil (HMO) and 20\% hydrogenated vegetable oil (HVO) which was mostly palm oil. Softer margarines which have beçome more popular in the United Kingdom in recent years were found to contain on average $30 \%$ hydrogenated vegetable oil, mainly soya, and only about $5 \%$ HMO. The rest of the material was natural unhydrogenated land-animal or vegetable fat which made up about $30 \%$ of the hard margarines and $65 \%$ of the soft margarines. Amounts of land-animal fat in UK margarines are small, about $15 \%$ on average.

Marine oils hardened to margarine specifications (HMO) contain, according to source, between $30 \%$ and $50 \%$ of $\mathrm{C}_{20}$ and $\mathrm{C}_{22}$ acids, largely mono-enoic. These acids are present in traditional fat sources, but at a low level. In HMO, transunsaturated acids form about $50 \%$ of the material, the greater part of which is unsaturated $C_{20}$ and $C_{22}$ acids.

Vegetable oils, in contrast, usually contain a negligible proportion of acids with carbon chains longer than $\mathrm{C}_{18}$ and when hydrogenated, between $12 \%$ and $25 \%$ of the fatty acids are transunsaturated acids, mostly 18:1. Rapeseed oil is exceptional as a vegetable oil in that it may contain up to $30 \%$ of erucic acid 22:1 w9 but this is entirely in the natural cis form. It is unlikely, however, that rapeseed oil is present in UK margarines in amounts greater than about $5 \%$.

Ruminant-animal fat (RAF) which includes total butter, beef, and mutton fats is characterised by the 
presence of certain fatty acids mostly with odd-number and branched chains. We grouped these together and defined the sum of acids 14:1, 15:0, 15:0 br, 15:1, 16:0 br, 17:0, and 17:1 as L. These form about $5 \%-8 \%$ of RAF, about $2 \%-3 \%$ of HMO, about $1 \%$ of pig and poultry fat, and are absent from HVO.

Transunsaturated acids are present in RAF in low proportions $(5 \%-7 \%)$ as a result of bacterial hydrogenation in the rumen, but they are absent from non-ruminant animal fats. These transunsaturated bonds in ruminant fats are usually more centrally situated in the carbon chain than they are in industrially hydrogenated fats, but we did not identify these separately in this study. Although the proportion of transunsaturated acids is low in RAF yet the consumption of RAF in Britain is three times greater than that of HF, so its contribution to total transacids $(T)$ is not negligible. Estimates of UK values in average dietary fat ${ }^{2}$ have been made as follows:

Trans acids from RAF

$2 \cdot 1 \%$

Trans acids from HF

$3 \cdot 3 \%$

Total trans acids (T)

$5 \cdot 4 \%$

$\mathrm{L}$ is much more characteristic of RAF but even in this group there may be some contribution from HF or from pig and poultry fat. Estimates of these ' $L$ ' acids in UK average fat are:

From RAF

From pig and poultry fat

From HF

$2 \cdot 2 \%$

$0.3 \%$

$0.3 \%$

Total (L)

$2 \cdot 8 \%$

Clearly, neither estimates of $\mathrm{T}$ or of $\mathrm{L}$ in isolation will yield unambiguous information, but we consider that the ratio $\mathrm{T}: \mathrm{L}$ might be more discriminating than either separately, and this ratio is central to our study. At the above relative consumptions of RAF and pig and poultry fat, the above ratio $T: L$ would be $2 \cdot 1: 2 \cdot 5=0 \cdot 8$ in the event of zero consumption of HF; in the estimated average UK diet it would be $5 \cdot 4: 2 \cdot 8=1 \cdot 9$ but as the proportion of HF increases, the ratio would increase still further.

Certain higher acids, 20:0, 20:1, 20:2, 20:3, 22:0 $22: 1$, which are typical of HMO, were grouped together as $\mathbf{H}$ and were analysed both as one group and separately.

We also examined the ratio of $(16: 0+16: 1) /(18: 0$ $+18: 1$ ) because this ratio, $R$, in RAF is about $0 \cdot 6$, in pig fat about $0 \cdot 5$, whereas in hydrogenated marine oil it varies between 1.1 and 1.4 according to source. Unfortunately, the majority of vegetable fats are low in $C_{16}$ and have values of $R, 0 \cdot 2$ to $0 \cdot 4$. $R$ therefore, although interesting, cannot discriminate between the fats under consideration.

We therefore decided to carry out a case-control study of the proportions of the various fatty acids in the body fat depots of decedents who had died of IHD compared with those who had died from other causes.

\section{Methods}

Adipose tissue specimens from the anterior wall near the umbilicus were supplied through the courtesy of the Medical Research Council Epidemiology Unit (South Wales). Cases consisted of male decedents dying from ischaemic heart disease (ICD 8th revision, categories 410 to 414 ); deaths in the same areas and of the same sex and similar age dying from unrelated causes were used as controls. All decedents were men who showed no evidence of wasting, and deaths from cerebrovascular disease or malignant neoplasms were excluded. The specimens, obtained by pathologists co-operating with the study at post-mortem examination, were transported in dry ice and stored in our laboratory in sealed containers at $-20^{\circ} \mathrm{C}$.

The proportions of the various fatty acids were measured by gas-liquid chromatography and the total of transunsaturated acids ( $T$ ) was measured by infrared spectroscopy. ${ }^{2}$

\section{Results}

A total of 231 specimens was available from 10 areas; 136 were from deaths attributed to ischaemic heart disease, and 95 from deaths attributed to the control group. The number of cases and controls in each area and the mean values for $L, T, T / L$ with their standard errors are shown in Table 1 . Corresponding mean values of $\mathrm{H}$ and 18:2 are as previously recorded. ${ }^{2}$

The age at death ranged between 31 and 63 years; mean ages at death for each of the 10 areas with their standard deviations are recorded in Table 1 . The overall mean for the 136 cases was 52.7 years and for the 95 controls 51.5 years. Regression coefficients for $T, L$, and $H$ respectively on age were +0.03 , +0.02 , and +0.003 units per year so it would appear safe to ignore this slight difference between mean ages.

We were able to obtain the social class status of 115 cases and 76 controls: $2 \%$ were in Social Class I, $12 \%$ in class II, $51 \%$ in class III, $25 \%$ in class IV, and $10 \%$ in class $\mathrm{V}$. The difference in status between cases and controls was slight; $12 \%$ of the cases and $17 \%$ of the controls were in classes I and II, 49\% and 54\% were in class III, and $39 \%$ and $29 \%$ were in classes IV and V ( $\chi^{2}$ with $2 \mathrm{df}=2 \cdot 44 \mathrm{p}>0.2$ ). 
Table 1 Mean and standard error of $T, L, T / L$ and ages at death, 136 cases versus 95 controls, for 10 areas or conurbations

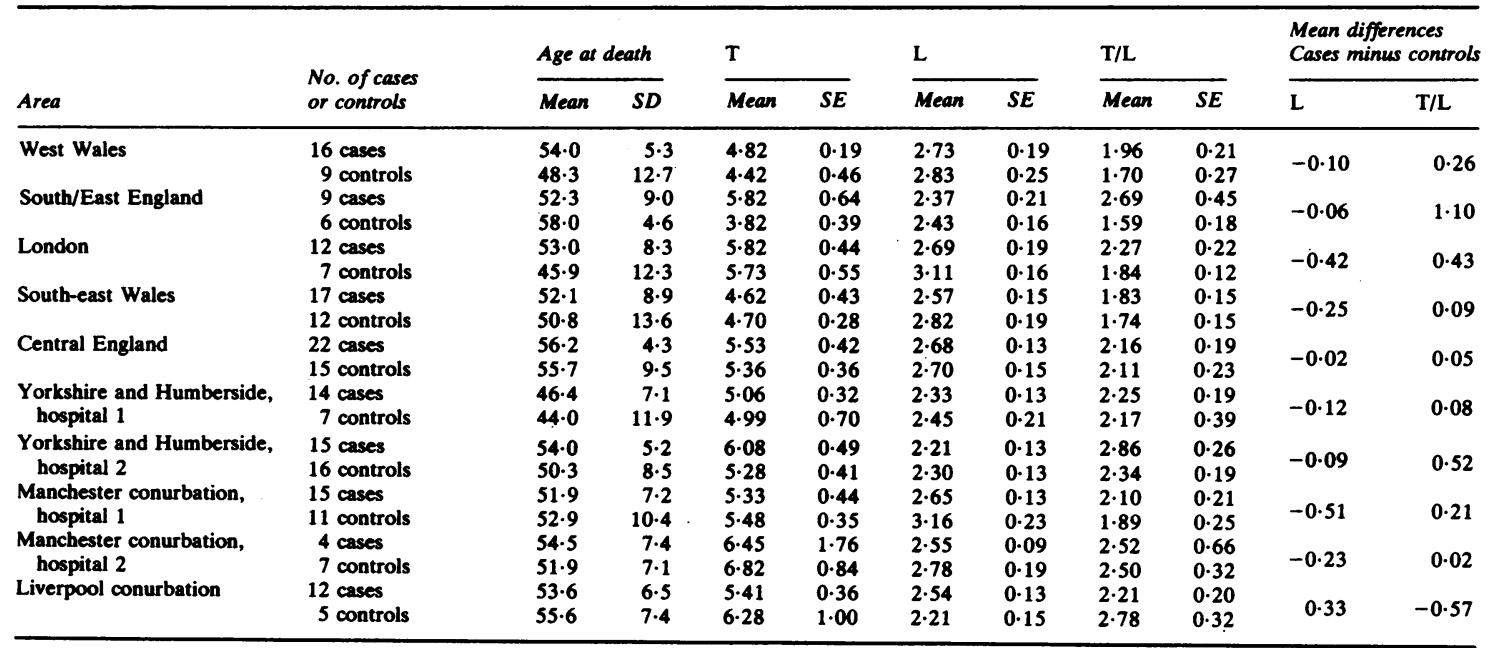

Analysis of variance in the controls shows that for $L$ the differences between areas are significant (Fisher's variance ratio $F=2.78, p<0.01$ ) and similarly for $T(F=2.40, p<0.025)$. Within each area, however, the differences between cases and controls are consistent and the mean value of $L$ is higher in the controls than in the cases in nine out of 10 areas. For $T / L$, in nine out of 10 areas the cases are higher than the controls.

The mean percentage of $\mathrm{T}$ for the control specimens in the study was $5 \cdot 2 \%$ and of $L$ it was $2 \cdot 7 \%$ which are close to the UK estimated values in average dietary fat.

The general pattern of regional variation in $\mathrm{T} / \mathrm{L}$ in the controls also broadly reflects known dietary patterns. HF consumption is lowest in the south, including Greater London and Wales; $T / L$ is lowest in the south-east, Greater London, and Wales; HF consumption is intermediate in the Midlands and $\mathrm{T} / \mathrm{L}$ is intermediate in central England; HF is highest in the north and north-west; $\mathrm{T} / \mathrm{L}$ is higher in Yorkshire and Humberside, Manchester and Liverpool. The consumption of RAF is in general the converse of that of HF. ${ }^{1}$

It would appear, therefore, that an adequate measure of matching for age, social class, and area of residence has been obtained.

We then applied the method of Pearce ${ }^{3}$ to weight blocks 'so that the experiment as a whole is made representative of the population' to which the results apply. Table 2 shows the weighted mean differences for cases minus controls of the proportion of the various fatty acids which we measured.

The greatest proportion of the adipose tissue composition was $18: 1$ at $44.9 \%$ and $16: 0$ at $22 \cdot 5 \%$
- Table 2 Overall adipose tissue composition; weighted differences cases minus controls

\begin{tabular}{|c|c|c|c|c|c|}
\hline \multirow{2}{*}{$\begin{array}{l}\text { Component } \\
\text { fatty } \\
\text { acids }\end{array}$} & \multirow{2}{*}{$\begin{array}{l}\% \\
\text { to } \\
\pm 0.05\end{array}$} & \multicolumn{4}{|c|}{ DIFFERENCES } \\
\hline & & $\begin{array}{l}\text { Mean } \\
\text { differences }\end{array}$ & $\begin{array}{l}\text { Standard } \\
\text { errors }\end{array}$ & $\begin{array}{l}\text { Student's } \\
t\end{array}$ & $\mathbf{P}$ \\
\hline $\begin{array}{l}10: 0+12: 0 \\
14: 0 \\
\text { L } \\
16: 0 \\
16: 1 \\
17: 0 \text { br } \\
18: 0 \\
18: 1 \\
18: 2 \\
H \\
18: 3 \\
20: 4\end{array}$ & $\begin{array}{c}0.6 \\
3.85 \\
2.6 \\
22.5 \\
7.15 \\
0.6 \\
4.95 \\
44.95 \\
8.05 \\
3.85 \\
0.65 \\
0.25 \\
100.0\end{array}$ & $\begin{array}{r}-0.037 \\
0.075 \\
-0.153 \\
0.506 \\
0.202 \\
0.004 \\
-0.312 \\
-0.266 \\
-0.014 \\
-0.007 \\
\ddagger \\
\ddagger\end{array}$ & $\begin{array}{l}0.043 \\
0.108 \\
0.079 \\
0.222 \\
0.227 \\
0.019 \\
0.156 \\
0.318 \\
0.278 \\
0.144\end{array}$ & $\begin{array}{r}-0.86 \\
0.70 \\
-1.93 \\
2.28 \\
0.89 \\
0.23 \\
-2.00 \\
-0.84 \\
-0.05 \\
-0.05\end{array}$ & $\begin{array}{l}\text { NS } \\
\text { NS } \\
\sim 0.05 \\
<0.025 \\
\text { NS } \\
\text { NS } \\
<0.05 \\
\text { NS } \\
\text { NS } \\
\text { NS }\end{array}$ \\
\hline $\begin{array}{l}\mathbf{T} \\
\mathrm{T} / \mathrm{L} \\
\mathbf{R} \\
\mathbf{M}\end{array}$ & $\begin{array}{c}5.33 \\
2 \cdot 16 \\
0.595 \\
55 \cdot 76\end{array}$ & $\begin{array}{c}0.226 \\
0.222 \\
0.0206 \\
-0.135\end{array}$ & $\begin{array}{l}0.218 \\
0.112 \\
0.0073 \\
0.427\end{array}$ & $\begin{array}{r}1.04 \\
1.98 \\
2.83 \\
-0.32\end{array}$ & $\begin{array}{l}\text { NS } \\
<0.05 \\
<0.01 \\
\text { NS }\end{array}$ \\
\hline
\end{tabular}

$\mathrm{L}=14: 1,15: 0,15: 0 \mathrm{br}, 16: 0 \mathrm{br}, 17: 0,15: 1,17: 1$

$\mathbf{H}=20: 0,20: 1,20: 2,20: 3,22: 0,22: 1$

$M=16: 1+18: 1+0.44 L+0.65 H$

$R=(16: 0+16: 1) /(18: 0+18: 1)$

$T=$ Total transunsaturated acids per cent.

₹ Previously shown² to be identical for case and control specimens within the limits of experimental error.

followed by $18: 2$ and $16: 1$; L, typical of RAF, formed $2.6 \%$ and the higher acids, $\mathrm{H}, 3.8 \%$. The total transunsaturated acids, $\mathrm{T}$, of all types were $5 \cdot 3 \%$ and the $T: L$ ratio was $2 \cdot 16$.

The differences between the proportions in cases and controls was statistically significant $(p<0.05)$ for 16:0 of which cases had a higher proportion and for 18:0 of which controls had a higher proportion. 
The difference in mean $\mathrm{L}$ was on the border of significance and although the difference in $\mathrm{T}$ was not significant the difference in mean $T / L$ was so.

\section{Discussion}

In the UK only about half of the consumption of HF is derived from retail margarines and hydrogenated cooking fats; the other half is derived from cakes, biscuits, pastries, and potato crisps or similar products bought as such. This has the effect of reducing individual variation in consumption of $\mathrm{HF}$ and so there is unlikely to be a large difference between cases and controls. Nevertheless, despite the small numbers involved and the slight differences seen, it is possible to come to some general conclusions.

Let us first consider the proportion of $L$ in the specimens, which is to an extent indicative of RAF. The mean proportion of $L$ was higher in the specimens from controls than in those from cases. This difference was on the margin of conventional significance $(t=1.93 p \sim 0.05)$. One explanation is that the controls consumed a higher proportion of RAF in their total fat intake from all sources. A second possibility is that the controls and cases consumed similar amounts of RAF, but that the excess $L$ in the controls was derived from HMO. It should be remembered that vegetable fats have zero L content whether hydrogenated or not. But the ratio T:L for HMO is around 15 compared with about unity for RAF. So if the controls had consumed a higher proportion of HMO they would also have had a large excess of $T$. In fact, however, the mean value of $T / L$ for the controls $(2.03)$ is significantly lower $(t=1.98, p<0.05)$ than for the cases $(2.25)$ so the excess $L$ could not have derived from HMO.

A third possibility is that the consumption by the two populations of both RAF and HMO is similar but that the excess $L$ in the control population is due to higher consumption of pig and poultry fat. However, since only $0.3 \%$ of the $2.8 \%$ of $L$ usually derives from pig and poultry fat, to remain consistent with the above difference in $T / L(0.22)$, the increased consumption would have to be substantial-over $100 \%$ higher-which seems very unlikely.

We conclude, therefore, that controls probably consumed on average a higher proportion of $L$ in their total dietary fat than did the cases, and that these acids derived essentially from RAF with possibly a minor contribution from non-ruminant animal fat.

Secondly, let us consider the total transunsaturated acids, $\mathrm{T}$, the proportion of which is lower for controls than for cases although the difference is not statistically significant $(t=1.04 p>0.05)$. These transunsaturated acids may be derived from RAF and from HF but since amounts in the former fat are lower than in the latter, a greater intake of HF relative to RAF should result in higher $T$. It is unlikely, therefore, that the controls would have a lower intake of RAF.

Thirdly, consider the ratio $R$. The mean proportion of $16: 0$ is significantly higher and the mean proportion of 18:0 significantly lower for the cases than for the control specimens, and this difference in molecular weights would be further accentuated if 16:1 and $18: 1$ were included. Consequently the ratio, $R=(16: 0+16: 1) /(18: 0+18: 1)$ is significantly higher for the cases $(t=2.83, p<0.01)$. Although this difference supports the view that the type of fat consumed might be implicated in the incidence of IHD, interpretation in terms of consumption of the various types considered in this paper is difficult. Thus whereas it is true that values of $R$ for RAF (around 0.6 ) and pig fat $(0.5)$ are substantially lower than the values for hydrogenated marine oils $(1 \cdot 1$ to 1.4 according to source), the majority of vegetable fats are low in $C_{16}$ and have very low values of $R(0 \cdot 2$ to 0.4 ) whether hydrogenated or not. It is clearly not possible to conclude from this result alone which material is responsible for the observed differences. It would, however, be difficult to argue in view of these ratios that the observed higher value of $R$ in the cases could be due to a higher consumption of animal fat (ruminant plus pig fat) and lower consumption of HF; if this were so, we would also expect $L$ to be higher for the cases, yet the mean value of $L$ is higher for the control group.

Fourthly, although we have found the proportion of $18: 2$ in depot fat to vary between $3.7 \%$ and $15.8 \%$, the weighted mean value for cases is virtually identical with that for the controls. This result lends no support to the currently popular view that higher amounts of polyunsaturated acids (essentially 18:2) which are present in many of the softer margarines have had a beneficial effect. It should be remembered, however, that in the UK until about 1974 consumption of soft margarines 'high in polyunsaturated acids' was quite small. In the American diet, according to Call and Sanchez, intake of polyunsaturated fatty acids almost doubled during the period 1940 to 1976 , and it seems very likely that a further increase has occurred since then. The main source of 18:2 in the British diet remains pig and poultry fat. ${ }^{2}$

Fifthly, the weighted means for the longer chain acids, $H$, are virtually identical for cases and controls, and therefore we have no evidence that 'higher acids' 
at the level consumed in the UK are harmful in themselves. In view of the claims by several authors that erucic acid, and probably 20:1 cis, are associated with damage to heart muscle, and that long-chain $\mathrm{C}_{20}$ to $\mathrm{C}_{24}$ saturated acids are atherogenic, a breakdown of our variable $\mathrm{H}$ into its components would seem desirable.

In fact our analyses show that the higher acid composition of depot fat is remarkably constant from area to area and between cases and controls for each of the 'higher' acids 20:0, 20:1, 20:2, 22:0, and 22:1. The one explanation for this might be that the differences of composition from one marine source to another cancel out with time due to the technical requirement to produce blended products of consistent physical properties.

Our results therefore show no differences between cases and controls for any particular higher acid, and do not lend any support to the above claims. But it should be added that, with the possible exception of 20:1, the individual components are present in very small amounts: thus 22:1 and 22:0 each make up on average somewhat less than $1 \%$ of the total fatty acid content of adipose tissue. Again, about $90 \%$ of higher marine unsaturated acids is in the transconfiguration, unlike the cis 22:1 and cis 20:1 of rapeseed oil.

Finally, the degree of mono-unsaturation is determined principally by the proportion of $16: 1$ plus 18:1 which together account for $52 \%$ of the total spectrum. There are also minor contributions from the mono-enoic content of 'higher acids' (65\%) and L (44\%). The figure for total degree of mono-unsaturation is seen to be virtually identical for case and control specimens.

A curious feature is that for case and control specimens, 16:0 content is positively correlated with 16:1 and negatively with 18:0 contents. It appears therefore that a self-regulating mechanism is involved which tends to buffer the overall degree of unsaturation. The implication of this result is at present under consideration.
We conclude, therefore, that there were differences in the type of body fat between cases who had died from arteriosclerotic disease and controls who died of other causes, and that these differences suggest that the cases may have consumed a higher proportion of hydrogenated fats and a lower proportion of ruminant-animal fat than did the controls.

We thank Dr. P. C. Elwood and the staff of the MRC Epidemiology Unit (South Wales) for obtaining appropriate case and control specimens; Professor O. L. Davies, Department of Statistics, University College of Wales, Aberystwyth, for critical appraisal of our statistical calculations; and Dr. Elspeth Smith, Department of Chemical Pathology, University of Aberdeen, and Professor H. Campbell, Department of Medical Statistics, Welsh National School of Medicine, for helpful suggestions. We also thank J. A. Winter for help in preparing the paper. We received a grant from the Welsh Committee for Locally Organised Research.

Reprints from Dr. Leo $H$. Thomas, Reader, Department of Science, the Polytechnic of Wales, Pontypridd, Mid Glamorgan CF37 1DL.

\section{References}

${ }^{1}$ Thomas LH. Mortality from arteriosclerotic disease and consumption of hydrogenated oils and fats. Br J Prev Soc Med 1975; 29: 82-90.

${ }^{2}$ Thomas LH, Jones PR, Winter JA, Smith $H$. Hydrogenated oils and fats; the presence of chemically-modified fatty acids in human adipose tissue. Am J Clin Nutr 1981; 34: 877-86.

${ }^{3}$ Pearce SC. The estimation of treatment means by designed experiments. Appl Stat 1974; 23: 22-5.

-Call DL, Sanchez AM. Trends in fat disappearance in the United States, 1909-65. J Nutr 1967; suppl, part II; 93: 1-28. 